\title{
APLICACIÓN DE UNA METODOLOGÍA PARA EVALUAR LA AGRESIVIDAD DEL AGUA HACIA ESTRUCTURAS DE CONCRETO
}

\author{
Asdrúbal Vargas ${ }^{1}$ \& José Francisco Fernández ${ }^{2}$ \\ ${ }^{1}$ Centro Servicio Exploración Subterránea \\ ${ }^{2}$ Centro Servicio Estudios Básicos de Ingeniería \\ Instituto Costarricense de Electricidad \\ E-Mail: avargass@ice.go.cr / jffernandez@ice.go.cr
}

\begin{abstract}
The chemical characteristics of surface and groundwater were analyzed near the main structure of the Toro III hydroelectric project. The reaction of water with concrete was determinated using the index of Langelier and Ryznar, which can be used to describe the aggressiveness of water over structures of concrete and city monuments constructed with sensible materials. Investigation of chemical characteristics and water aggressiveness to concrete is necessary in projects where the water is frequently in contact with the walls of the tunnel, similar to the Toro III project. The water samples from embalse Toro II show high concentrations of major ions; the samples from Gemelas and Flor Creek have negative values of Langelier index $(<-1,5)$. All these samples are aggressive to concrete. The water samples collected in Flor creek have very low concentrations of major ions as comparedto the rest of the samples.
\end{abstract}

\begin{abstract}
RESUMEN: Se pretende analizar la agresividad química de las aguas superficiales de varias quebradas que discurren cercanas al trazado del túnel del proyecto hidroeléctrico Toro III y clasificar las aguas de acuerdo al diagrama de Stiff que permite identificar variaciones en las concentraciones de los elementos. La concentración de la mayoría de elementos analizados resultó más alta en las muestras de agua recolectadas en el embalse de la planta Toro II, la cual será utilizada en el proyecto Toro III. Los análisis químicos realizados a las muestras de la quebrada Flor presentan concentraciones menores comparados con el resto de las muestras analizadas. En cuanto al valor de índice de Langelier, los valores más negativos se presentan en la quebrada Gemelas y le sigue la quebrada Flor. Todas las muestras analizadas son agresivas al concreto, pues los índices de Langelier obtenidos son inferiores a $-1,5$.
\end{abstract}

\section{INTRODUCCIÓN}

La importancia del conocimiento y la aplicación de las metodologías de análisis hidrogeoquímicos se aprecia con la construcción de túneles revestidos de concreto, presas de tipo CCR (concreto compactado con rodillo) y vertederos de excedencias.

El objetivo de este trabajo es estudiar las características químicas de las aguas superfi- ciales en varias quebradas que se encuentran dentro del área de influencia del proyecto hidroeléctrico Toro III y utilizar el índice de Langelier para caracterizar la agresividad del agua hacia el concreto. El proyecto hidroeléctrico Toro III (Fig. 1), se encuentra bajo la influencia climática del Caribe con una estación lluviosa prolongada. Además, se ubica en terrenos volcánicos de pendientes moderadas, limitada al Oeste por la fila Chocosuela y al Este por la 


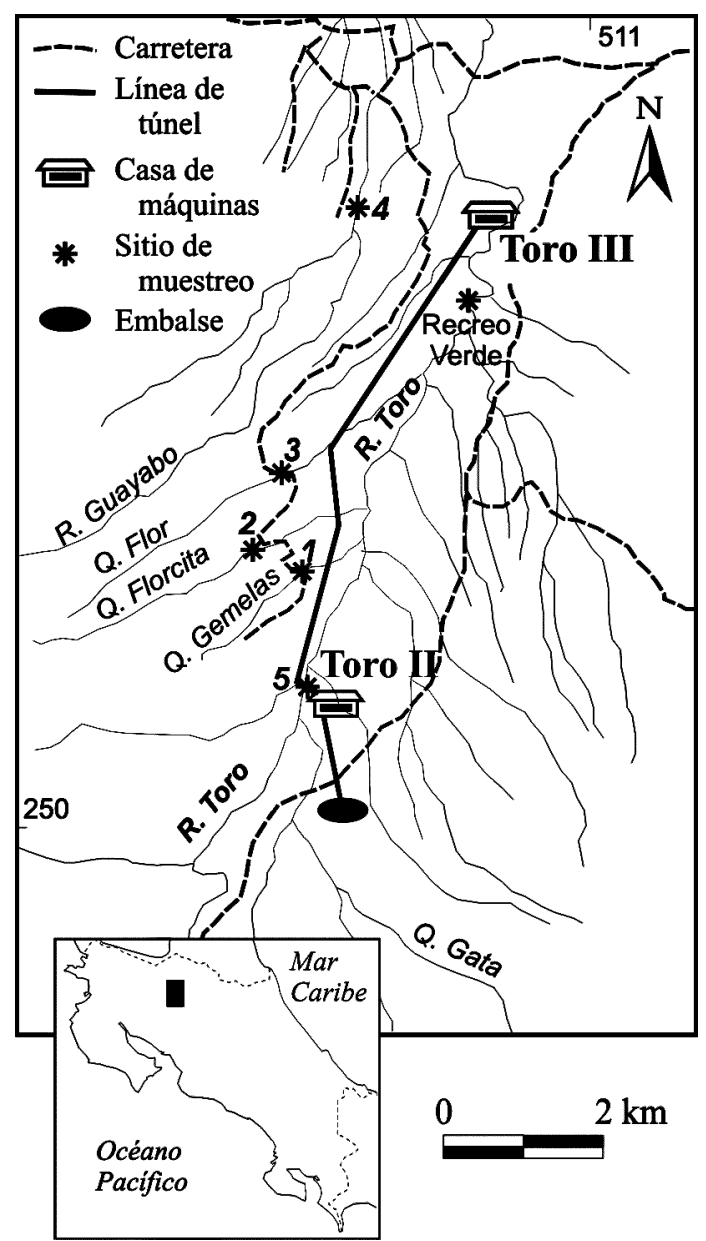

Fig. 1: Mapa de ubicación de la zona de estudio del proyecto Toro III (parte de las hojas topográficas Quesada y Poás del IGN).

Fila El Gorrión. (Madrigal, 1999). Hacia el río Toro descargan numerosos afluentes, entre ellos las quebradas Flor, Florcita y Gemelas. El río Guayabo discurre, dentro del área del proyecto, paralelo al río Toro quien tiene una dirección Suroeste hacia el Noreste.

Se ha considerado conveniente estudiar el proyecto Toro III, ya que se cuenta con información que permite demostrar la aplicación de índices para evaluar la agresividad del agua hacia el concreto en una zona caracterizada por la acidez en varios ríos y quebradas. Para realizar este trabajo se cuenta con los resultados de los análisis químicos mensuales de aguas superfi- ciales correspondientes a los años 1999, 2000 y algunos meses del 2001 de las quebradas Flor, Florcita, Gemelas, río Guayabo y del embalse del proyecto Toro II.

La técnica empleada en la recolección de muestras de agua fue la de muestreo simple instantáneo, según las normas "Standard Methods for Examination of Water and Wastewater" (Anónimo, 1985). Se utilizaron envases de polietileno de $1000 \mathrm{ml}$. En el caso del muestreo de agua superficial de ríos o quebradas, las botellas se cerraron bajo la superficie del agua o de la manera más rápida posible con el objeto de aislar por completo las muestras de agua del anhídrido carbónico atmosférico. Tanto la recolección como el análisis de las muestras estuvo a cargo del personal del laboratorio químico del Centro de Servicio de Estudios Básicos de Ingeniería del ICE.

La metodología de análisis ha consistido en la integración de los datos del índice de Langelier en un diagrama de variación temporal, ya que es posible que se presenten variaciones dependiendo de la época de invierno o verano. Además se procedió a la construcción de diagramas de Stiff para evaluar las variaciones de los elementos químicos, que permitan definir posibles unidades hidroquímicas. Debido a que en la zona existen evidencias de que el agua de algunos ríos es agresiva hacia el concreto y perjudicial para las obras que estén en contacto con este tipo de agua es de suma importancia evaluar las características químicas de las quebradas y ríos que tendrían interacción directa con el túnel y otras estructuras del proyecto.

\section{ATAQUE DEL AGUA HACIA EL CONCRETO Y EFECTO SOBRE OBRAS CIVILES}

El ataque del agua hacia el concreto puede originarse por medio de aguas blandas, sulfatos, cloruros, ácidos o por un ataque combinado de varios agentes (Cuadro 1). Es posible medir la agresividad, por medio del índice de saturación de Langelier. 
Cuadro 1

Grado de ataque de varios parámetros

\begin{tabular}{lccc}
\hline Compuesto químico & Débil & Fuerte & Muy Fuerte \\
\hline Ácidos $(\mathrm{pH})$ & $6,5-5,5$ & $5,5-4,5$ & $<4,5$ \\
Ácido carbónico $\left(\mathrm{CO}_{2}\right)$ en mg/L & $15-30$ & $30-60$ & $>60$ \\
Magnesio $\left(\mathrm{Mg}^{+2}\right)$ en $\mathrm{mg} / \mathrm{L}$ & $100-300$ & $300-1500$ & $>1500$ \\
Sulfato $\left(\mathrm{SO}_{4}^{-2}\right)$ en mg/L & $200-600$ & $600-3000$ & $>3000$ \\
\hline
\end{tabular}

Fuente: Mason (1989).

\section{Aguas blandas}

El agua que drena desde regiones montañosas es muchas veces de bajo contenido de sales disueltas. Presenta valores de $\mathrm{pH}$ cercanos a 7 , pero es agresiva al concreto, cuando tiende a disolver el hidróxido de calcio $\left(\mathrm{Ca}(\mathrm{OH})_{2}\right)$, presente en el cemento para formar carbonato de calcio. El proceso de descomposición es marcadamente acelerado por la presencia de $\mathrm{CO}_{2}$ disuelto en agua blanda, cuando se encuentra en exceso del que se requiere para convertir el hidróxido de calcio a carbonato de calcio. El exceso de dióxido de carbono reacciona con el agua para formar ácido carbónico, el cual reacciona con el carbonato de calcio para formar bicarbonato de calcio $\left(\mathrm{Ca}\left(\mathrm{HCO}_{3}\right)_{2}\right)$. Al contrario del carbonato de calcio, el bicarbonato de calcio es muy soluble en agua y es fácilmente removido. Al considerar el grado de ataque el $\mathrm{pH}$ es un factor importante pero no puede ser tomado como una simple medida de la agresividad. Es necesario tomar en cuenta parámetros como la temperatura, alcalinidad, contenido de los sólidos totales, contenido de calcio y el contenido de dióxido de carbono (Mason, 1989).

\section{Cloruros}

Los cloruros en altas concentraciones constituyen otro factor que representa agresividad hacia el concreto. Los iones cloruro aumentan la microfisuración, es decir, facilitan la formación de poros por donde penetra el agua, favoreciendo los procesos de carbonatación. Sin embargo, el efecto más significativo de la presencia de los cloruros en alta concentración es, atacar las estructuras de hierro y acero que se encuentran en el interior de las estructuras de concreto o en lugares expuestos favoreciendo el proceso de picado ("pitting") y la corrosión. Pero la presencia de cloruros podría reducir el efecto del ataque de los sulfatos, mecanismo que no está definido claramente (Mason, 1989).

\section{Sulfatos}

El ataque de los sulfatos al concreto puede darse debido a la alta concentración de este en el agua. El concreto, cuando es atacado por agua con altas concentraciones de sulfatos, presenta un aspecto hinchado con una superficie blanca y pastosa. La agresividad del sulfato depende del tipo de catión que lo constituye. El más nocivo corresponde al sulfato de amonio, en orden descendente le sigue el de magnesio, de sodio y finalmente el de calcio. El ataque de los sulfatos se presenta como una reacción química de los sulfatos con el hidróxido de calcio o los aluminatos de calcio y las ferritas del cemento, provocando una recristalización y el aumento de volumen antes mencionado y finalmente una disgregación por fracturación a menor escala, provocando la destrucción del cemento. A continuación se describen algunas reacciones que se llevan a cabo en los componentes del cemento. El sulfato de magnesio, por ejemplo, ejerce una acción agresiva porque reacciona con el $\mathrm{Ca}(\mathrm{OH})_{2}$ y también con los otros componentes impuros del calcio formando una pasta de cemento hidratada de acuerdo a las siguientes reacciones. 


$$
\begin{gathered}
\mathrm{Ca}(\mathrm{OH})_{2}+\mathrm{MgSO}_{4}+2 \mathrm{H}_{2} \mathrm{O}=\mathrm{CaSO}_{4} 2 \mathrm{H}_{2} \mathrm{O}+ \\
\mathrm{Mg}(\mathrm{OH})_{2} \\
3 \mathrm{CaO} \cdot \mathrm{Al}_{2} \mathrm{O}_{3} 6 \mathrm{H}_{2} \mathrm{O}+3 \mathrm{MgSO}_{4}=3 \mathrm{CaSO}_{4}+ \\
2 \mathrm{Al}(\mathrm{OH})_{3}+3 \mathrm{Mg}(\mathrm{OH})_{2} \\
3 \mathrm{CaO} \cdot 2 \mathrm{SiO}_{2 \text { (acuoso) }}+3 \mathrm{MgSO}_{4 \text { (acuoso) }}=3 \mathrm{CaSO}_{4} \\
+\underset{\mathrm{Mg}_{(\mathrm{OH})_{2}}}{+} 2 \mathrm{SiO}_{2 \text { (acuoso) }}
\end{gathered}
$$

El sulfato de calcio que se obtiene por la reacción del $\mathrm{MgSO}_{4}$ con impurezas del aluminato hidratado, forma un compuesto que provoca expansión en la pasta de cemento. El sulfato de sodio reacciona también con el hidróxido de calcio según la reacción:

$$
\begin{gathered}
\mathrm{Ca}(\mathrm{OH})_{2}+\mathrm{Na}_{2} \mathrm{SO}_{4}+\underset{\mathrm{NaOH}}{2 \mathrm{H}_{2} \mathrm{O}}=\mathrm{CaSO}_{4} 2 \mathrm{H}_{2} \mathrm{O}+ \\
2 \mathrm{O}
\end{gathered}
$$

El sulfato de sodio, es problemático porque el nuevo compuesto forma cristales de mayor tamaño. También reacciona con el aluminato de calcio formando el sulfoaluminato de calcio como se indica en la siguiente reacción:

$$
\begin{gathered}
3\left(4 \mathrm{CaO} \cdot \mathrm{Al}_{2} \mathrm{O}_{3} \cdot 13 \mathrm{H}_{2} \mathrm{O}\right)+6 \mathrm{Na}_{2} \mathrm{SO}_{4}= \\
2\left(3 \mathrm{CaO} \mathrm{Al} \mathrm{O}_{3} 3 \mathrm{CaSO}_{4} 31 \mathrm{H}_{2} \mathrm{O}\right)+ \\
2 \mathrm{Al}(\mathrm{OH})_{3}+12 \mathrm{NaOH}
\end{gathered}
$$

El sulfato de calcio presente en el agua deteriora el cemento a causa de la reacción entre el sulfato y los aluminatos de calcio hidratado del cemento endurecido. La siguiente reacción ilustra lo que sucede en este caso:

$$
\begin{gathered}
3 \mathrm{CaO} \mathrm{Al} \mathrm{O}_{3} \cdot 6 \mathrm{H}_{2} \mathrm{O}+3 \mathrm{CaSO}_{4}+26 \mathrm{H}_{2} \mathrm{O}= \\
3 \mathrm{CaO} \mathrm{Al}_{2} \mathrm{O}_{3} \cdot 3 \mathrm{CaSO}_{4} \cdot 32 \mathrm{H}_{2} \mathrm{O}
\end{gathered}
$$

El agua tiende a hidrolizar el trisulfoaluminato y descomponerlo según la reacción:

$3 \mathrm{CaO} \cdot \mathrm{Al}_{2} \mathrm{O}_{3} 3 \mathrm{Ca} \mathrm{SO}_{4} 32 \mathrm{H}_{2} \mathrm{O}+\mathrm{H}_{2} \mathrm{O}=$ $3 \mathrm{CaSO}_{4}+3 \mathrm{Ca}(\mathrm{OH})_{2}+2 \mathrm{Al}(\mathrm{OH})_{3}+\mathrm{H}_{2} \mathrm{O}$

De lo anterior se deduce que las condiciones para que el concreto experimente ataque por $\mathrm{CaSO}_{4}$ son las siguientes: a) alta concentración de iones sulfato en el agua

b) contenido de $\mathrm{Ca}(\mathrm{OH})_{2}$ y aluminato hidratado en la pasta de cemento relativamente alta.

Por otro lado el ataque de los sulfatos se puede traducir como una descomposición de los silicatos de calcio producto de las reacciones con estos, provocando una disminución de la resistencia del concreto.

\section{Ácidos}

El agua con ácidos libres podría disolver el cemento y los agregados conteniendo carbonatos y, probablemente, tendría lugar la corrosión de aceros, que constituyen vigas y estructuras de concreto.

\section{Índice de saturación de Langelier}

Para estimar la agresividad del agua se utiliza el índice de Langelier, en el cual se toman en cuenta el potencial de hidronio $(\mathrm{pH})$, la concentración de calcio, la alcalinidad, la temperatura y la concentración de sólidos disueltos en la muestra de agua. El cálculo del índice se puede realizar con la siguiente expresión:

$$
\begin{aligned}
& \mathrm{IL}=\mathrm{pH}+\log \mathrm{C}+\log \mathrm{A}+0,025 \mathrm{~T}-0,011 \mathrm{~S}^{1 / 2} \\
& -12,30
\end{aligned}
$$

\section{Donde}

IL: Índice de Langelier

$\mathrm{pH}$ : potencial de ion hidronio

C: Dureza temporal, concentración de calcio expresado como $\mathrm{CaCO}_{3}$ en unidades de $\mathrm{mg} / \mathrm{l}$.

A: Alcalinidad expresado como $\mathrm{CaCO}_{3}$ en unidades de $\mathrm{mg} / \mathrm{l}$.

$\mathrm{T}$ : Temperatura en grados celcius, en un rango de 0 a $25{ }^{\circ} \mathrm{C}$.

$\mathrm{S}$ : Sólidos totales disueltos ( $\mathrm{mg} / \mathrm{l})$, donde $\mathrm{S}$ es menor que $1000 \mathrm{mg} / \mathrm{l}$ 
A partir del índice de Langelier calculado y utilizando el cuadro 2 es posible clasificar el agua según los grados de agresividad.

Cuadro 2

Agresividad del agua según índice de Langelier

\begin{tabular}{cl}
$\begin{array}{c}\text { Indice de } \\
\text { Langelier }\end{array}$ & \multicolumn{1}{c}{ Efecto } \\
\hline$>0$ & $\begin{array}{l}\text { No hay agresión, posible incrustación y } \\
\text { deposición de calcio } \\
\text { Agresiva } \\
0-1,5\end{array}$ \\
$<-1,5$ & Muy agresiva, concreto puede ser atacado
\end{tabular}

\section{Posibles efectos del agua en las estructuras de concreto}

Entre los efectos que podrían producir los agentes antes mencionados se cuentan la pérdida de resistencia del concreto, las pérdidas de estabilidad en las presas por una reducción de la cohesión y la corrosión del acero de refuerzo entre otros. Además, cuando ocurre lixiviación de los concretos y de las lechadas en las pantallas de inyección, se produce un incremento de la permeabilidad que aumenta la infiltración y los sólidos disueltos en el agua (Rojas et. al, 1990).

\section{CARACTERÍSTICAS HIDROQUÍMICAS EN EL P.H. TORO III}

El análisis del agua superficial en el PH Toro III se realizó en la quebrada Gemelas (punto 1), Florcita (punto 2), Flor (punto 3), río Guayabo (punto 4) y en el embalse del proyecto Toro II (punto 5). Los sitios de muestreo se pueden accesar por medio de la carretera que conduce desde Venecia de San Carlos hasta la casa de Máquinas del proyecto hidroeléctrico Toro II. El agua de las quebradas Flor, Florcita, Las Gemelas y el río Guayabo está siendo muestreada de manera regular en los puntos definidos en la figura 1 .

En la quebrada Flor se analizaron 24 muestras de enero 1999 a febrero 2001 (Cuadro 3). Los

Cuadro 3

Valores de características químicas del agua

\begin{tabular}{|c|c|c|c|c|c|c|c|c|c|c|c|c|c|c|c|}
\hline & pHL & $\mathrm{Na}^{+}$ & $\mathrm{K}^{+}$ & $\mathrm{Ca}^{+2}$ & $\mathrm{Mg}^{+2}$ & $\mathrm{Fe}^{+3}$ & $\mathrm{SiO}_{2}$ & SD & ST & $\mathrm{PO}_{4}^{3-}$ & $\mathrm{Cl}-$ & $\mathrm{SO}_{4}^{-2}$ & $\mathrm{HCO}^{3-}$ & DT & IL \\
\hline \multicolumn{16}{|c|}{ Q. Flor } \\
\hline Máx. & 7,64 & 1,67 & 0,60 & 3,64 & 1,62 & 0,10 & 25,80 & 84,00 & 88,00 & 0,18 & 1,77 & 1,19 & 26,50 & 16,40 & $-2,09$ \\
\hline Prom. & 7,07 & 1,38 & 0,51 & 2,72 & 1,27 & 0,05 & 17,04 & 31,54 & 36,58 & 0,04 & 1,02 & 0,66 & 19,83 & 11,74 & $-2,79$ \\
\hline Mín. & 6,26 & 0,93 & 0,37 & 1,94 & 0,97 & 0,01 & 11,20 & 8,00 & 20,00 & 0,01 & 0,72 & 0,28 & 13,90 & 0,86 & $-3,67$ \\
\hline \multicolumn{16}{|c|}{ Q. Florcita } \\
\hline Máx. & 7,94 & 2,11 & 0,80 & 5,06 & 1,81 & 0,08 & 29,70 & 156,0 & 194,0 & 0,33 & 1,35 & 1,96 & 32,00 & 23,60 & $-1,46$ \\
\hline Prom. & 7,37 & 1,85 & 0,66 & 4,43 & 1,54 & 0,03 & 22,15 & 45,50 & 51,79 & 0,06 & 0,99 & 0,76 & 27,95 & 17,08 & $-2,17$ \\
\hline Mín. & 6,69 & 1,34 & 0,54 & 3,27 & 1,20 & 0,01 & 13,20 & 12,00 & 18,00 & 0,01 & 0,48 & 0,30 & 22,40 & 1,20 & $-2,80$ \\
\hline Desv. & 0,33 & \multicolumn{13}{|c|}{ Q. Gemelas } & 0,38 \\
\hline Máx. & 7,60 & 1,70 & 0,64 & 6,07 & 1,35 & 0,09 & 26,90 & 118,0 & 136,0 & 0,61 & 2,16 & 1,74 & 31,90 & 17,60 & $-2,11$ \\
\hline Prom. & 7,11 & 1,41 & 0,54 & 2,56 & 1,06 & 0,05 & 18,74 & 33,58 & 38,29 & 0,09 & 1,05 & 0,80 & 18,09 & 10,88 & $-2,86$ \\
\hline Mín. & 6,52 & 1,07 & 0,42 & 1,78 & 0,77 & 0,01 & 11,20 & 6,00 & 4,00 & 0,02 & 0,76 & 0,22 & 13,00 & 0,92 & $-3,41$ \\
\hline \multicolumn{15}{|c|}{ Río Guayabo } & 0,37 \\
\hline Máx. & 8,16 & 3,54 & 1,19 & 7,74 & 4,62 & 0,13 & 38,00 & 218,0 & 394,0 & 0,22 & 1,81 & 1,79 & 45,40 & 35,60 & $-0,88$ \\
\hline Prom. & 7,53 & 2,57 & 0,91 & 5,90 & 1,95 & 0,04 & 26,14 & 59,33 & 71,62 & 0,07 & 1,32 & 1,11 & 33,79 & 21,63 & $-1,79$ \\
\hline Mín. & 6,41 & 1,57 & 0,57 & 4,06 & 1,10 & 0,02 & 15,10 & 14,00 & 16,00 & 0,01 & 0,40 & 0,11 & 24,60 & 1,34 & $-3,06$ \\
\hline \multicolumn{6}{|c|}{ Embalse Toro II } & 0,03 & $\begin{array}{c}5,86 \\
\text { Embal }\end{array}$ & $\begin{array}{l}40,52 \\
\text { e Toro I }\end{array}$ & 75,94 & 0,04 & 0,37 & 0,42 & 5,77 & 6,64 & 0,47 \\
\hline Máx. & 7,40 & 5,50 & 2,50 & 13,60 & 3,90 & 1,00 & 53,70 & 137,0 & 138,0 & 0,09 & 6,19 & 30,90 & 28,60 & 50,60 & $-1,98$ \\
\hline Prom. & 7,22 & 3,81 & 1,94 & 9,93 & 2,95 & 0,17 & 38,13 & 103,2 & 108,3 & 0,08 & 4,23 & 20,62 & 24,29 & 38,29 & $-2,17$ \\
\hline Mín. & 6,96 & 1,90 & 1,12 & 5,10 & 1,48 & 0,01 & 21,00 & 52,00 & 64,00 & 0,05 & 1,76 & 3,95 & 19,60 & 16,80 & $-2,57$ \\
\hline Desv. & 0,13 & 1,08 & 0,42 & 2,47 & 0,73 & 0,31 & 10,25 & 27,67 & 25,38 & 0,02 & 1,41 & 9,74 & 3,23 & 9,88 & 0,20 \\
\hline
\end{tabular}

(pHL: potencial de hidronio determinado en el laboratorio, SD: sólidos disueltos, ST: sólidos totales, DT: dureza total, IL: índice de Langelier). Valores en $\mathrm{mg} / \mathrm{L}$ excepto $\mathrm{pH}$ e IL. 
valores del índice de Langelier tienen un valor promedio de $-2,79$ y un potencial del ion hidronio con 7,07. Se observan variaciones temporales del índice, que varía entre $-3,7$ y $-2,1$, y se nota un incremento del índice durante la época de invierno y una disminución en los meses de diciembre a marzo (Fig. 2). Los valores más bajos se presentaron en noviembre de 1999 y enero de 2001, debido a una baja concentración de calcio, bicarbonato y un $\mathrm{pH}$ bajo.

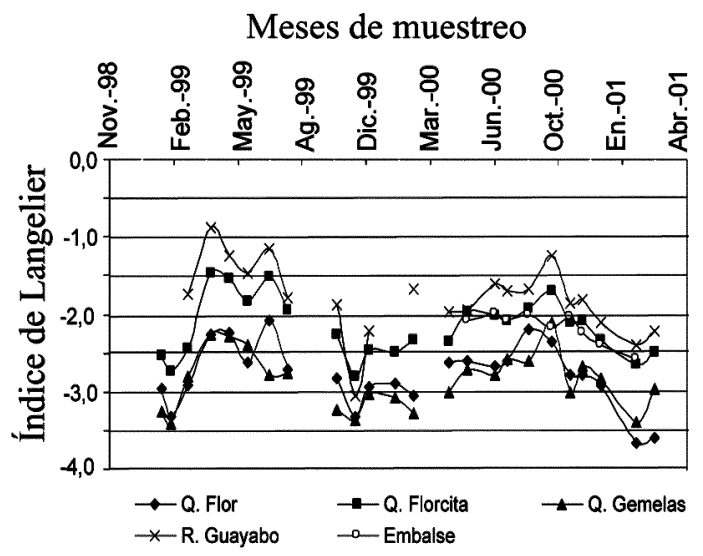

Fig. 2: Variación temporal del índice de Langelier para las muestras de las quebradas analizadas en el proyecto Toro III.

Se tienen 24 análisis de agua en la quebrada Florcita de enero 1999 hasta febrero 2001. El valor promedio del índice de Langelier es de $-2,2$ varíando de $-2,8$ a $-1,5$, mostrándose el incremento del índice durante los meses de la época lluviosa y un descenso del mismo en los meses de verano (Fig. 2). El valor más bajo del índice se presentó en noviembre de 1999. El potencial de hidronio varía de 6,7 a 7,9 (Cuadro 3).
Se dispone de 24 análisis de agua en la quebrada Gemelas correspondientes al período de enero 1999 hasta febrero 2001. Presentan un valor promedio para el índice de Langelier de $-2,86$ y los valores máximos y mínimos alcanzados varían de $-2,11$ a $-3,41$ respectivamente (Cuadro 3), mostrando un incremento a principios de la época lluviosa y un descenso a principios de la época seca (Fig. 2) similar a las quebradas Flor y Florcita.

Se tienen 21 análisis entre marzo de 1999 y febrero del 2001 en el río Guayabo. Los valores del índice de Langelier presentan una tendencia descendente desde marzo de 1999 hasta diciembre, punto a partir del cual crece de manera irregular el valor hasta octubre del $2000 \mathrm{y}$ finalmente desciende hasta marzo 2001. Las muestras de agua del río Guayabo presentan un índice promedio de $-1,79$, un valor mínimo de $-3,06$ y un máximo de $-0,88$. Por su parte el $\mathrm{pH}$ varía de 6,4 a 8,2 (Cuadro 3).

Aunque solo se cuentan con datos de Junio 2000 a Febrero 2001 provenientes del embalse de Toro II se aprecia una tendencia descendente del índice de Langelier (Fig. 2). El valor promedio del índice es de $-2,17$, el valor máximo es de $-1,98$ y el valor mínimo es de 2,57 (Cuadro 3).

En el sitio Recreo Verde (Fig. 1) existen manantiales termales en la margen izquierda del río Toro que se caracterizan por un $\mathrm{pH}$ bajo y la elevada concentración de la mayoría de iones mayores sobre todo bicarbonatos y magnesio, aunque son notorias las variaciones de una muestra a otra. Se tienen tres muestras de agua del 18 de setiembre de 1999 a partir de las cuales se han analizado 16 parámetros (cuadro 4).

Resultados químicos de los manantiales Recreo Verde (mg/l excepto el pH)

\begin{tabular}{|c|c|c|c|c|c|c|c|c|c|c|c|c|c|c|c|c|}
\hline & pHL & $\mathrm{HCO}^{3-}$ & $\mathrm{Na}^{+}$ & $\mathrm{K}^{+}$ & $\mathrm{Ca}^{+2}$ & $\mathrm{Mg}^{+2}$ & $\mathrm{SiO}_{2}$ & $\mathrm{~F}^{-}$ & $\mathrm{Cl}^{-}$ & $\mathrm{NO}^{3-}$ & $\mathrm{SO}_{4}^{-2}$ & $\mathrm{PO}_{4}^{3-}$ & $\mathrm{Mn}^{+2}$ & $\mathrm{Cu}^{+2}$ & $\mathrm{Zn}^{+2}$ & $\mathrm{Fe}^{+3}$ \\
\hline M1 & 6,56 & 2517,0 & 474,0 & 140,0 & 246,0 & 424,0 & 125,0 & 0,12 & 510,0 & 1,31 & 883,0 & 0,09 & 0,12 & 0,04 & 0,02 & 0,02 \\
\hline M2 & 6,57 & 1583,0 & 304,0 & 65,8 & 209,0 & 256,0 & 98,0 & 0,22 & 306,0 & 0,90 & 469,0 & 0,06 & 0,24 & 0,03 & 0,02 & 0,02 \\
\hline M3 & 4,58 & 13,5 & 8,94 & 3,18 & 15,5 & 7,80 & 37,4 & 1,35 & 25,3 & 0,28 & 44,2 & 0,18 & 0,13 & 0,03 & 0,02 & 0,14 \\
\hline
\end{tabular}




\section{DISCUSIÓN DE RESULTADOS}

La comparación del agua muestreada en los diferentes puntos permite realizar la siguiente clasificación en cuanto a agresividad hacia el concreto de acuerdo al índice de Langelier para las muestras provenientes del proyecto Toro III.

Q. Gemelas $(-2,86)>$ Q. Flor $(-2,79)>\mathrm{Q}$. Florcita $(-2,17)>$ embalse Toro II $(-2,17)>\mathrm{R}$. Guayabo $(-1,79)$. De acuerdo al índice de Langelier, el agua en todos los sitios es agresiva al concreto. Esto ocurre, pues el agua contiene ciertos compuestos que podrían reaccionar con los constituyentes del cemento provocando expansión y fisuración.

La variación del $\mathrm{pH}$ incide directamente en el índice de Langelier, ya que este parámetro tiene un peso mayor que los otros; por lo tanto, este índice debería ser considerado un indicador que sugiere una tendencia hacia depositar o disolver carbonato de calcio. El grado de ataque del $\mathrm{pH}$ no puede ser tomado como una simple medida de la agresividad. Es necesario tomar en cuenta la temperatura, alcalinidad, el contenido de los sólidos totales, contenido de calcio y el contenido de dióxido de carbono. Aún si el agua presenta un $\mathrm{pH}$ neu- tro, con baja concentración de sales disueltas y bicarbonato; es muy probable que ocurra el proceso de lixiviación.

La concentración de la mayoría de parámetros estudiados es mayor en el embalse de Toro II debido al aporte de agua proveniente del río Desagüe (Cuadro 5). La quebrada Flor muestra los valores más bajos en la mayoría de parámetros. En cuanto al índice de Langelier, los valores más negativos se presentan en la quebrada Gemelas y le sigue la quebrada Flor. Las muestras analizadas son agresivas al concreto, pues los índices de Langelier obtenidos son inferiores a $-1,5$.

Las concentraciones de varios parámetros y el valor del índice de Langelier varían en función principalmente de la cantidad de lluvia de la zona y a la escorrentía asociada. Para mostrar esta dependencia con respecto a la lluvia se han obtenido datos de precipitación de la estación Quebrada Gata (Fig. 3). El índice de Langelier más bajo ocurre en Noviembre 1999 y en enero 2001 en correspondencia con meses muy lluviosos en la zona.

Por medio de los diagramas de Stiff se aprecia que las concentraciones de sulfatos, calcio y bicarbonatos de las muestras del embalse de Toro II son mayores que en los otros puntos de muestreo (Fig. 4). Por otro lado, los manantiales

Cuadro 5

Comparación de parámetros físico-químicos para los sitios de muestreo

\begin{tabular}{|c|c|c|c|c|c|}
\hline & Queb. Flor & Queb. Florcita & Queb. Gemelas & Río Guayabo & Embalse Toro II \\
\hline $\mathrm{pH}$ & 7,07 & 7,37 & 7,11 & 7,53 & 7,22 \\
\hline $\mathrm{Na}^{+}(\mathrm{mg} / \mathrm{l})$ & 1,38 & 1,85 & 1,41 & 2,57 & 3,81 \\
\hline $\mathrm{K}^{+}(\mathrm{mg} / \mathrm{l})$ & 0,51 & 0,66 & 0,54 & 0,91 & 1,94 \\
\hline $\mathrm{Ca}^{+}(\mathrm{mg} / \mathrm{l})$ & 2,72 & 4,43 & 2,56 & 5,90 & 9,93 \\
\hline $\mathrm{Mg}^{2+}(\mathrm{mg} / \mathrm{l})$ & 1,27 & 1,54 & 1,06 & 1,95 & 2,95 \\
\hline $\mathrm{Fe}^{3+}(\mathrm{mg} / \mathrm{l})$ & 0,05 & 0,03 & 0,05 & 0,04 & 0,17 \\
\hline $\mathrm{SiO}_{2}(\mathrm{mg} / \mathrm{l})$ & 17,04 & 22,15 & 18,74 & 26,14 & 38,13 \\
\hline $\mathrm{SD}(\mathrm{mg} / \mathrm{l})$ & 31,54 & 45,50 & 33,58 & 59,33 & 103,20 \\
\hline $\mathrm{ST}(\mathrm{mg} / \mathrm{l})$ & 36,58 & 51,79 & 38,29 & 71,62 & 108,3 \\
\hline $\mathrm{PO}_{4}^{-}(\mathrm{mg} / \mathrm{l})$ & 0,04 & 0,06 & 0,09 & 0,07 & 0,08 \\
\hline $\mathrm{Cl}^{-}(\mathrm{mg} / \mathrm{l})$ & 1,02 & 0,99 & 1,05 & 1,32 & 4,23 \\
\hline $\mathrm{SO}_{4}^{-}(\mathrm{mg} / \mathrm{l})$ & 0,66 & 0,76 & 0,80 & 1,11 & 20,62 \\
\hline $\mathrm{HCO}_{3}^{-}(\mathrm{mg} / \mathrm{l})$ & 19,83 & 27,95 & 18,09 & 33,79 & 24,29 \\
\hline $\mathrm{DT}(\mathrm{mg} / \mathrm{l})$ & 11,74 & 17,08 & 10,88 & 21,63 & 38,29 \\
\hline IL & $-2,79$ & $-2,17$ & $-2,86$ & $-1,79$ & $-2,17$ \\
\hline
\end{tabular}

(pHL: potencial de hidronio de laboratorio, SD: sólidos disueltos, ST: sólidos totales, DT: dureza total, IL: índice de Langelier). 


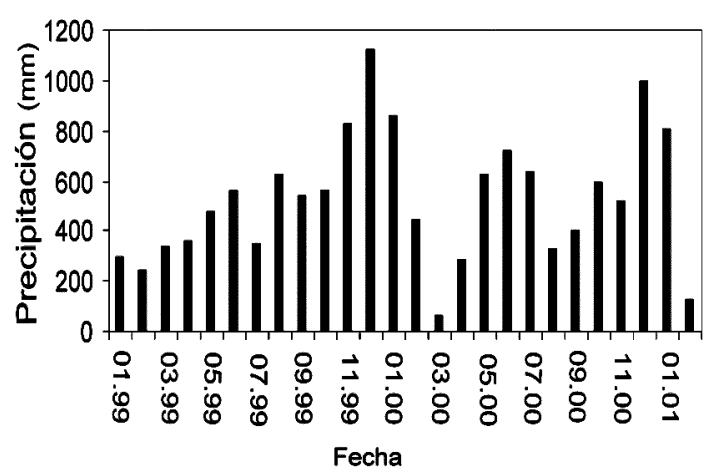

Fig. 3: Precipitación mensual en la estación quebrada Gata para los años 1999 y 2000; notándose que alcanza un máximo en noviembre y diciembre.

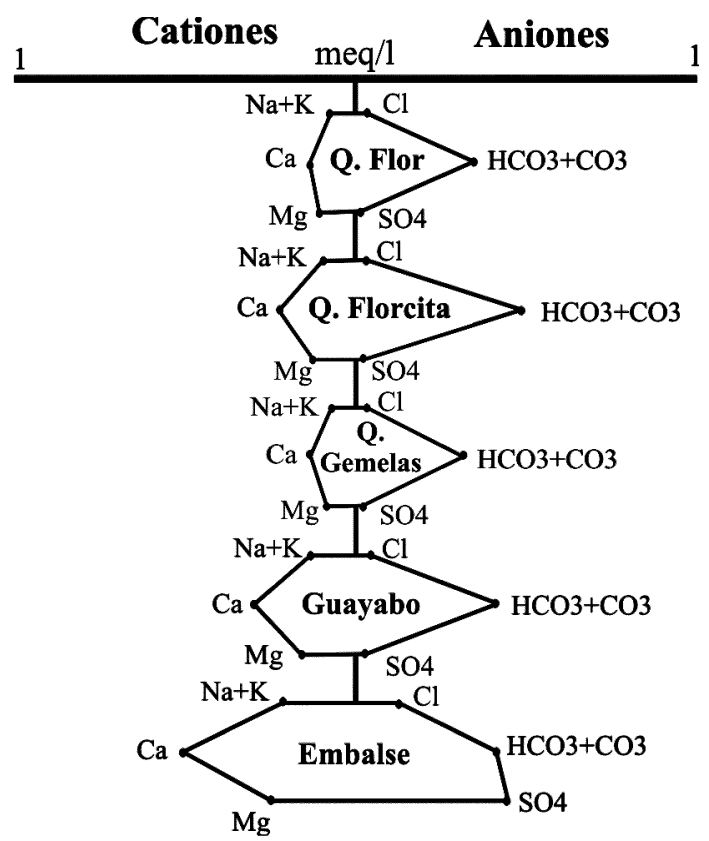

Fig. 4: Diagramas de Stiff para las muestras del proyecto Toro III. Las muestras de agua del embalse Toro II tienen mayor concentración comparadas con el resto de las muestras.

de Recreo Verde son enriquecidos en bicarbonato y magnesio. Las muestras de los sitios Flor, Florcita, Guayabo y Gemelas son de tipo bicarbonatadas mixtas, pero con tendencia a cálcicas. Esto se debe a la litología presente en la zona, como lavas de tipo andesítico, que contienen pla- gioclasa y augita que permiten la liberación del ión calcio; la presencia del bicarbonato se debe a la disolución de dióxido de carbono atmosférico o a alguna fuente geotermal.

\section{RECOMENDACIONES}

Para la construcción del túnel del P.H. Toro III se recomienda estudiar la composición del agua subterránea en el área de estudio y evaluar el índice de Langelier para calcular su agresividad hacia el concreto. Además, se sugiere utilizar cementos puzolánicos que serán aplicados en las zonas donde el túnel pasará cerca de la quebrada Florcita y Gemelas. Así mismo, debe tomarse en cuenta que el agua proveniente del embalse de Toro II, que será utilizada en el proyecto Toro III, es agresiva al concreto según el índice de Langelier. La relación de agua - cemento debe ser baja para formar una masa cementante de baja permeabilidad e impedir el ataque del agua a través de fisuras. Es importante realizar ensayos de agresión sobre muestras de concreto utilizando agua proveniente de las quebradas y ríos estudiados, así como de perforaciones.

\section{REFERENCIAS}

ANÓNIMO, 1985: Standard methods for the examination of water and wastewater. - 1268 págs. [16 ${ }^{\mathrm{a}}$ ed.] APHA, AWWA, WPCF. Washington.

MASON, P. J., 1989: Exposure of dam concrete to special aggressive waters. - 177 págs. ICOLD, Paris.

MADRIGAL, C., 1999: Informe geológico de prefactibilidad del proyecto hidroeléctrico Toro III. - 20 págs. Tomo I. Área de Ingeniería Geológica, ICE, San José [Inf. interno].

ROJAS, L., LEÓN, C., VEGA, O., ESTRADA, J., \& FERNÁNDEZ, J.F., 1990: Estudio de la caracterización físico-química de las aguas naturales en la cuenca alta del río Toro y su influencia con las obras del proyecto. - 54 págs. Dirección de planificación eléctrica. ICE, San José [Inf. interno]. 